\title{
Re-evaluating the potential of immunoprophylaxis and/or immunotherapy for infections caused by multidrug resistant Klebsiella pneumoniae
}

\author{
Scott D Kobayashi ${ }^{1}$ \& Frank R DeLeo*,1 \\ ${ }^{1}$ Laboratory of Bacteriology, Rocky Mountain Laboratories, National Institute of Allergy \& Infectious Diseases, National Institutes of \\ Health, Hamilton, MT, 59840, USA \\ *Author for correspondence: fdeleo@niaid.nih.gov
}
' there is the potential to substantially reduce the incidence of infection and the dependence on antibiotics"

First draft submitted: 20 June 2018; Accepted for publication: 28 June 2018; Published online:

26 September 2018

Keywords: antibiotic resistance $\bullet$ antibody $\bullet$ carbapenem $\bullet$ innate immunity $\bullet$ vaccine

Antimicrobial resistance is a global health problem that poses significant hurdles to prevent and treat infectious diseases. The emergence of antibiotic-resistant bacterial pathogens contributes to increased morbidity and mortality, prolonged hospital stays and a greater financial burden on the healthcare system. In addition, antibiotic resistance increases the risk of medically invasive procedures, and effective antimicrobial therapy is confounded by continual emergence of novel mechanisms of resistance. Perhaps one of the most feared consequences of antibiotic resistance is the emergence of pan-resistant strains for which there are either limited or no available treatment options. Such bacterial strains have emerged within the past 10 years, and many of these strains are Klebsiella spp.

Klebsiella are relatively ubiquitous Gram-negative bacteria within the family Enterobacteriaceae. These microbes are present in the environment, including soil, plants and water. Some species, such as Klebsiella pneumoniae, are also commensal organisms of farm animals and humans [1]. Klebsiella spp. are carried asymptomatically in approximately $30 \%$ of the human population, primarily in the intestine [1]. Given the normal high burden of colonization in humans, it is not surprising that Klebsiella are opportunistic pathogens and a significant cause of healthcare-associated infections. For example, Magill et al. reported that $K$. pneumoniae and $K$. oxytoca account for a combined total of $9.9 \%$ of all healthcare-associated infections, which ranks third among all organisms recovered [2]. These data were obtained from 183 hospitals reporting to the National Healthcare and Safety Network in 20092010 [2]. In those studies, Klebsiella infections were primarily associated with the urinary tract, surgical sites and the bloodstream, and they caused pneumonia. The high incidence of Klebsiella infections in hospitals is compounded by antibiotic resistance.

The ubiquitous nature of Klebsiella spp. brings them into contact with many other bacterial species, thereby facilitating significant uptake and/or exchange of genetic material, a process known as horizontal gene transfer. Wyres and Holt estimated recently that Klebsiella as a species has acquired more than 400 genes encoding antimicrobial resistance elements, far more than other Gram-negative human pathogens of notable clinical concern [3]. Gram-negative bacteria that produce extended-spectrum $\beta$-lactamases (ESBLs), including Escherichia coli and $K$. pneumoniae, emerged in the late 1980s and 1990s and became a major problem in hospitals. These microbes are resistant to extended-spectrum cephalosporin and monobactam antibiotics [4], important therapeutics for the treatment of infections caused by Gram-negative bacteria. In addition, such strains often harbor resistance to other important antibiotics, such as fluoroquinolones. Therefore, carbapenem antibiotics became the therapy of choice for infections caused by ESBL-producing organisms [4].

For most antibiotics, emergence of resistance is simply a matter of time (e.g., see https://www.cdc.gov/drugresi stance/about.html). Indeed, carbapenem-resistant K. pneumoniae were first reported in the USA by Yigit $e$ t al. in 
2001 [5], and the pathogen has emerged worldwide since the mid-2000s [6]. Carbapenem resistance is conferred largely by $K$. pneumoniae carbapenemase (KPC) [5] or New Delhi metallo- $\beta$-lactamase 1 (NDM-1), either of which confers resistance to virtually all $\beta$-lactam antibiotics. The most abundant strains in the USA produce KPC and are also resistant to fluoroquinolone and aminoglycoside antibiotics $[7,8]$. As much as these multidrug-resistant strains are difficult to treat, there is a high mortality rate associated with bacteremia [9] and new treatments and/or prophylactics are needed.

Given the high mortality rate of individuals with bacteremia caused by KPC strains, it would be remiss to not evaluate all alternative nonantibiotic therapeutic options. To this end, it is evident that the requirement for alternatives to antibiotics is clearly recognized and serious consideration has been given to alternative approaches including $\mathrm{mAb}$ therapy and active vaccination schemes [10]. In short, a vaccine would provide a viable option to prevent and treat infections with antimicrobial-resistant organisms, and there is the potential to substantially reduce the incidence of infection and the dependence on antibiotics (and slow the emergence of resistant organisms). There are currently no licensed vaccines available for treatment or prevention of $K$. pneumoniae.

\section{Passive immunization}

The microbial surface is decorated with carbohydrates such as capsular polysaccharides and O-antigens of lipopolysaccharide, that have been exploited as successful candidate antigens for vaccination strategies. Antimicrobial resistance has renewed interest in vaccine development and recent investigation of these historic antigenic targets. There are at least seven distinct Klebsiella LPS serotypes defined by unique O-antigen structures. In a survey of 709 clinical isolates, Pennini et al. [11] recently reported that serotype O1 was predominant in antibiotic susceptible strains, and carbapenem-resistant strains (including ST258) are predominantly serotype O2. Human monoclonal antibodies raised against both $\mathrm{O} 1$ and $\mathrm{O} 2$ antigens were protective in mouse models of $K$. pneumoniae bacteremia and pneumonia, and administration of $\mathrm{mAbs}$ provided synergistic adjunctive protection with antibiotics (carbapenem). The ability of anti-K. pneumoniae LPS-O-antigen mAbs to enhance opsonophagocytic killing by neutrophils rather than neutralize stimulation by LPS is important in protection against pneumonia and lethal infection [12].

A K. pneumoniae lineage classified as multilocus sequence type 258 (ST258) is present in hospitals in many regions of the world, including the USA [7,8,13]. Based on genomic analyses of clinical isolates, ST258 is comprised of two major genetic lineages or clades [14]. Notably, these clades are differentiated by a hypervariable region (a region of DNA recombination) that contains genes-encoding enzymes for biosynthesis of capsule polysaccharide. ST258 clinical isolates can be identified readily by PCR analysis as capsule polysaccharide type 1 (cps-1) or type 2 (cps-2), or some other capsule type [15]. Clinical isolates that harbor $c p s-1$ (clade 1) produce CPS1, and those that contain $c p s-2$ (clade 2) produce CPS2. These two capsule types are produced by the majority of ST258 clinical isolates in the USA and elsewhere $[8,16]$. Historically, passive immunization using antibody raised against $K$. pneumoniae CPS has been shown to be both effective in animal models of infection and safe for administration in humans. More recently, Diago-Navarro et al. [17] investigated the utility of anti-CPS mAbs raised against the K1-serotype predominant in hypermucoid strains of $K$. pneumoniae. The mAbs demonstrated protective efficacy against $K$. pneumoniae in murine models of sepsis and pneumonia, and intravital microscopy indicated they promote efficient uptake by phagocytic cells. CPS is a prominent virulence attribute of $K$. pneumoniae and known to inhibit uptake and killing by phagocytic cells and provide protection against complement-mediated cell lysis. K. pneumoniae ST258 that are ingested by neutrophils are killed efficiently [18]. Polyclonal antibodies directed against $K$. pneumoniae CPS2 readily promote opsonophagocytosis and enhance killing by human neutrophils and complement [19]. In addition, murine mAbs raised against CPS2 promote intra- and extracellular killing of $K$. pneumoniae and are protective in a mouse model of infection [17].

\section{Active immunization}

Previous studies investigated active immunization against $K$. pneumoniae, including use of killed whole cell preparations, cell lysates, proteins and purified polysaccharides. One of the most successful vaccine attempts was a 24-valent Klebsiella CPS vaccine, and although promising and safe, the vaccine was ultimately halted due to incomplete strain coverage and production difficulties. Bacterial capsular polysaccharides present a unique challenge for vaccine development, as they are thymus-independent antigens, are poor activators of $\mathrm{T}$ cells and do not lead to generation of $\mathrm{T}$ and B-cell memory. Fortunately, improvements in technology and advances in our understanding of immunology facilitate new approaches. Immunogenicity and efficacy of carbohydrate vaccines can be successfully enhanced 
by coupling CPS to carrier proteins, typically inactivated bacterial toxins. This approach has been successful for development of vaccines to other encapsulated bacterial pathogens and the vast majority of glycoconjugate vaccines licensed thus far are CPS and CPS derivatives. Recently, Seeberger et al. described a semisynthetic glycoconjugate candidate vaccine for carbapenem-resistant $K$. pneumoniae [20]. The vaccine comprised of a hexasaccharide repeating unit and associated substructures covalently linked to the diphtheria toxin-based carrier CRM197. The CRM197based vaccine was immunogenic in both mice and rabbits and produced antibodies that were cross-reactive with K. pneumoniae CPS. The semisynthetic glycoconjugate vaccine induced a high level of IgG1 antibodies and the antibodies were opsonophagocytic in differentiated HL-60 cells [20]. The ability of the vaccine to protect against $K$. pneumoniae challenge was not assessed due in large part to the absence of a good experimental model that approximates the natural history of human infection in susceptible patients.

\section{Conclusion}

The antibiotic resistance era has rapidly reached a point where the antimicrobial pipeline has become perilously thin, and the problem forces healthcare to the precipice of disaster. There is clearly a need to consider alternative strategies to combat infections caused by resistant microbes. For $K$. pneumoniae, the ideal vaccine would have universal coverage against a diversity of strains including those that are carbapenem resistant. To date there is no magic bullet - no vaccine strategy has emerged with enough coverage to potentially overcome low enthusiasm due to poor marketability. It is encouraging that there is significant experimental evidence that a vaccine approach is possible to combat disease caused by pan-resistant strains of $K$. pneumoniae. Moving forward, it is clear that in order to improve the current approaches there is significant need to develop appropriate experimental models that simulate at-risk populations.

\section{Financial \& competing interests disclosure}

The authors are supported by the Intramural Research Program of the National Institute of Allergy and Infectious Diseases, National Institutes of Health. The authors have no other relevant affiliations or financial involvement with any organization or entity with a financial interest in or financial conflict with the subject matter or materials discussed in the manuscript apart from those disclosed.

No writing assistance was utilized in the production of this manuscript.

\section{References}

1. Brisse S, Grimont F, Grimont, P.A.D. The Genus Klebsiella. In: The Prokaryotes, Dworkin M, Falkow S, Rosenberg E, Schleifer K-H, Stackebrandt E (Eds). Springer Science+Business Media, LLC, New York, NY, USA, 159-196 (2006).

2. Magill SS, Edwards JR, Bamberg W et al. Multistate point-prevalence survey of health care-associated infections. N. Engl. J. Med. 370(13), 1198-1208 (2014).

3. Wyres KL, Holt KE. Klebsiella pneumoniae as a key trafficker of drug resistance genes from environmental to clinically important bacteria. Curr. Opin. Microbiol. 45, 131-139 (2018).

4. Pitout JD, Laupland KB. Extended-spectrum beta-lactamase-producing Enterobacteriaceae: an emerging public-health concern. Lancet Infect. Dis. 8(3), 159-166 (2008).

5. Yigit H, Queenan AM, Anderson GJ et al. Novel carbapenem-hydrolyzing beta-lactamase, KPC-1, from a carbapenem-resistant strain of Klebsiella pneumoniae. Antimicrob. Agents Chemother. 45(4), 1151-1161 (2001).

6. Munoz-Price LS, Poirel L, Bonomo RA et al. Clinical epidemiology of the global expansion of Klebsiella pneumoniae carbapenemases. Lancet Infect. Dis. 13(9), 785-796 (2013).

7. Kitchel B, Rasheed JK, Patel JB et al. Molecular epidemiology of KPC-producing Klebsiella pneumoniae isolates in the United States: clonal expansion of multilocus sequence type 258. Antimicrob. Agents Chemother. 53(8), 3365-3370 (2009).

8. Peirano G, Bradford PA, Kazmierczak KM, Chen L, Kreiswirth BN, Pitout JD. Importance of clonal complex 258 and IncFK2-like plasmids among a global collection of Klebsiella pneumoniae with blaKPC. Antimicrob. Agents Chemother. 61(4), e02610-16 (2017).

9. Falagas ME, Tansarli GS, Karageorgopoulos DE, Vardakas KZ. Deaths attributable to carbapenem-resistant Enterobacteriaceae infections. Emerg. Infect. Dis. 20(7), 1170-1175 (2014).

10. Czaplewski L, Bax R, Clokie M et al. Alternatives to antibiotics-a pipeline portfolio review. Lancet Infect. Dis. 16(2), 239-251 (2016).

11. Pennini ME, De Marco A, Pelletier M et al. Immune stealth-driven O2 serotype prevalence and potential for therapeutic antibodies against multidrug resistant Klebsiella pneumoniae. Nat. Commun. 8(1), 1991 (2017).

12. Cohen TS, Pelletier M, Cheng L et al. Anti-LPS antibodies protect against Klebsiella pneumoniae by empowering neutrophil-mediated clearance without neutralizing TLR4. JCI Insight 2(9), e92774 (2017). 
13. Cerqueira GC, Earl AM, Ernst CM et al. Multi-institute analysis of carbapenem resistance reveals remarkable diversity, unexplained mechanisms, and limited clonal outbreaks. Proc. Natl Acad. Sci. USA 114(5), 1135-1140 (2017).

14. Deleo FR, Chen L, Porcella SF et al. Molecular dissection of the evolution of carbapenem-resistant multilocus sequence type 258 Klebsiella pneumoniae. Proc. Natl Acad. Sci. USA 111(13), 4988-4993 (2014).

15. Chen L, Chavda KD, Findlay J et al. Multiplex PCR for identification of two capsular types in epidemic KPC-producing Klebsiella pneumoniae sequence type 258 strains. Antimicrob. Agents Chemother. 58(7), 4196-4199 (2014).

16. Satlin MJ, Chen L, Patel G et al. Multicenter clinical and molecular epidemiological analysis of bacteremia due to carbapenem-resistant Enterobacteriaceae (CRE) in the CRE epicenter of the United States. Antimicrob. Agents Chemother. 61(4), e02349-16 (2017).

17. Diago-Navarro E, Motley MP, Ruiz-Perez G et al. Novel, Broadly reactive anticapsular antibodies against carbapenem-resistant Klebsiella pneumoniae protect from infection. MBio 9(2), e00091-18 (2018).

18. Kobayashi SD, Porter AR, Dorward DW et al. Phagocytosis and killing of carbapenem-resistant ST258 Klebsiella pneumoniae by human neutrophils. J. Infect. Dis. 213(10), 1615-1622 (2016).

19. Kobayashi SD, Porter AR, Freedman B et al. Antibody-mediated killing of carbapenem-resistant ST258 Klebsiella pneumoniae by human neutrophils. MBio 9(2), e00297-18 (2018).

20. Seeberger PH, Pereira CL, Khan N et al. A semisynthetic glycoconjugate vaccine candidate for carbapenem-resistant Klebsiella pneumoniae. Angew. Chem. Int. Ed. Engl. 56(45), 13973-13978 (2017). 